\title{
On the Asymptotic Behavior of Solutions of Semi-linear Wave Equations
}

By

\author{
Akitaka MATSUMURA*
}

\section{Introduction}

We first consider the following semi-linear wave equation in Part I:

$$
u_{t t}-\Delta u+m^{2} u+g \mid u !^{p-1} u=0
$$

where $m>0, g>0, p>1, x \in R^{n}, \Delta=$ Laplacian. Recently, Glassey [1] showed that if $p$ is small $\left(1<p \leqq 2 n=1 ; 1<p \leqq 1+2 n^{-1} n \geqq 2\right)$, scattering theory is impossible for complex solutions of (1). We show in 1.1 Glassey's result is also applicable even to real solutions. Segal showed in [3] and [4] that scattering operator can be constructed for (1) if $p>2+2 n^{-1}$. We show in 1.2 that the solution $u_{+}(x, t)$ of the free equation [(1) with $g=0]$ to which a given solution $u(x, t)$ of (1) is asymptotic in a weak sense as $t \rightarrow+\infty$, exists if $p>1+2 n^{-1}, n \geqq 3$.

In Part II, we consider the following semi-linear wave equation with the first order dissipation:

$$
u_{t t}-\Delta u+u_{t}+f\left(u, u_{t}, \nabla u\right)=0 .
$$

$\mathrm{W}$ e show the asymptotic properties of the solutions of the linear equation [(2) with $f=0]$ in 2.1 and those of the nonlinear equation (2) in 2.2 and 2.3 .

Notation. We denote by $L^{p}$ the space of measurable functions $u$ on $R^{n}$ whose $p$-th powers $(1 \leqq r \leqq \infty)$ are integrable with the norm

$$
\|u\|_{L^{p}}=\left(\int|u(x)|^{p} d x\right)^{1 / p}\|u\|_{\infty}=\mathrm{ess} \cdot \sup _{x}|u(x)|
$$

Communicated by S. Matsuura, November 11, 1975.

* Department of Applied Mathematics and Physics, Faculty of Engineering, Kyoto University. 
by $C^{\infty}$ the space of infinitely differentiable functions, by $C_{0}^{\infty}$ the subspace of $C^{\infty}$ consisting of functions with compact support in $R^{n}$ and by $H^{k}$ the usual Sobolev space on $R^{n}$ with the norm

$$
\|u\|_{k}^{2}=\sum_{|\alpha| \leqq k}\left\|\left(\frac{\partial}{\partial x}\right)^{\alpha} u\right\|_{L^{2}}^{2}
$$

where

$$
\left(\frac{\partial}{\partial x}\right)^{\alpha}=\frac{\partial^{|\alpha|}}{\partial x_{1}^{\alpha_{1}} \cdots \partial{x_{n}}^{\alpha_{n}}}, \quad|\alpha|=\alpha_{1}+\cdots+\alpha_{n} .
$$

Let $X$ be a Banach space on $R^{n}$. Then $\mathcal{E}_{t}{ }^{k}(X) \ni u(x, t)$ means that $u(\cdot, t)$ belongs to $X$ for all fixed $t$ and $u$ is $k$-times continuously differentiable with respect to $t$ in $X$-topology. We denote $\operatorname{grad} u=\left(\partial u / \partial x_{1}, \partial u / \partial x_{2}, \cdots\right.$, $\left.\partial u / \partial x_{n}\right)$ by $\nabla u$. In Part I, all functions are generally complex-valued, but in Part II, all functions are real-valued.

\section{Part I}

\section{§ 1. 1. Extention of Glassey's Result}

We consider the solutions of the Cauchy problem for the equation (1). We take $g=1$ without loss of generality. In [1], Glassey's result is not valid for real solutions, because he assumes

$$
Q(t)=\operatorname{Im} \int \bar{u} u_{t} d x \neq 0 .
$$

Then we define the momentum $P(t)$ by

$$
P(t)=\operatorname{Re} \int \bar{u}_{t} \nabla u d x
$$

instead of $Q(t)$. We denote the energy norm $\|\cdot\|_{e}$ by

$$
\|u(t)\|_{e}^{2}=\int\left(\left|u_{t}\right|^{2}+|\nabla u|^{2}+m^{2}|u|^{2}\right) d x .
$$

We have the following theorem which is valid for both real and complex solutions.

Theorem 1. Let $u(x, t)$ be a $C^{2}$-solution of equation (1) with Cauchy data in $C_{0}{ }^{\infty}$ satisfying $P(0) \neq 0$. Suppose that 


$$
1<p \leqq 2 \text { if } n=1,1<p \leqq 1+2 n^{-1} \text { if } n \geqq 2 \text {. }
$$

Then, there dose not exist any free solution $v(x, t)$ in $C_{0}^{\infty}$ such that

$$
\|u(t)-v(t)\|_{e} \rightarrow 0 \text { as } t \rightarrow+\infty .
$$

Proof. We note the following energy equality holds: if $u$ and $v$ are $C_{0}{ }^{2}$ solution of the nonlinear equation (1) and the free one respectively, it follows that

$$
\begin{aligned}
& \int\left(\left|u_{t}\right|^{2}+|\nabla u|^{2}+m^{2}|u|^{2}+\frac{2}{p+1}|u|^{p+1}\right) d x=\text { const. } \\
& \int\left(\left|u_{t}\right|^{2}+|\nabla v|^{2}+m^{2}|v|^{2}\right) d x=\text { const. for all } t \geqq 0 .
\end{aligned}
$$

We first show that $P(t)$ is a time invariant vector. Differentiating $P(t)$ with respect to $t$ directly,

$$
\begin{aligned}
\frac{d P(t)}{d t} & =\operatorname{Re}\left(\int \bar{u}_{t} \nabla u_{t} d x+\int \bar{u}_{t t} \nabla u d x\right) \\
& =\frac{1}{2} \int \nabla\left(\left|u_{t}\right|^{2}-|\nabla u|^{2}-m^{2}|u|^{2}-\frac{2}{p+1}|u|^{p+1}\right) d x \\
& =0 .
\end{aligned}
$$

Hence, we have

$$
P(t)=P(0) \text { for all } t \geqq 0 \text {. }
$$

Suppose that there exists a free solution $v \in C_{0}{ }^{\infty}$ such that

$$
\|u(t)-v(t)\|_{e} \rightarrow 0 \text {, as } t \rightarrow+\infty \text {. }
$$

Define

$$
P_{0}(t)=\operatorname{Re} \int \bar{v}_{t} \nabla v d x
$$

We note that $P_{0}(t)$ is also time invariant vector from the same argument as before. Then

$$
P(t)-P_{0}(t)=\operatorname{Re}\left\{\int\left(\bar{u}_{t}-\bar{v}_{t}\right) \nabla u+\bar{v}_{t}(\nabla u-\nabla v) d x\right\},
$$

by using Schwarz's inequality

$$
\text { | } P(t)-P o(t) i_{1}\|\nabla u\|_{L^{2}}\|u-v\|_{e}+\left\|v_{t}\right\|_{L^{2}}\|u-v\|_{e} .
$$


Since $\|\nabla u\|_{L^{2}}$ and $\left\|v_{t}\right\|_{L^{2}}$ are bounded from (3) and

$$
\|u(t)-v(t)\|_{e} \rightarrow 0 \text { as } t \rightarrow+\infty
$$

we have

$$
\left|P(t)-P_{0}(t)\right| \rightarrow 0 \text { as } t \rightarrow+\infty .
$$

Hence, from $P(t)-P o(t)=$ const., it follows

$$
P(t)=P_{0}(t)=P(0) \text { for all } t \geqq 0 \text {. }
$$

Then, the assumption $P(0) \neq 0$ gives

$$
\begin{aligned}
0<|P(0)| & =\left|\operatorname{Re} \int \bar{v}_{t} \nabla_{v} d x\right|=\left|-\operatorname{Re} \int v \nabla \bar{v}_{t} d x\right| \\
& \leqq\left\|v_{t}\right\|_{e}\|v\|_{L^{2}} .
\end{aligned}
$$

On the other hands, since $v_{t}$ is also free solution, we have from (3),

$$
\left\|v_{t}(t)\right\|_{e}=\left\|v_{t}(0)\right\|_{e} \text { for all } t \geqq 0 .
$$

Therefore, there is a positive constant $c_{0}$ such that

$$
\int|v(x, t)|^{2} d x \geqq c_{0} \quad \text { for all } t \geqq 0 .
$$

Let the data of the free solution $v$ be supported in the ball $|x| \leqq k$. Then, by the support property and Hölder's inequality, we have

$$
\begin{aligned}
0<c_{0} & \leqq \int|v(x, t)|^{2} d x=\int_{|x| \leqq x+t}|v(x, t)|^{2} d x \\
& \leqq\left(\int|v(x, t)|^{p+1} d x\right)^{2 /(p+1)}\left(\int_{|x| \leqq k+t} 1 d x\right)^{(p-1) /(p+1)} \\
& \leqq \text { const. } t^{(p-1) /(p+1) n}\left(\int|v(x, t)|^{p+1} d x\right)^{2 /(p+1)} \quad \text { for all } t \geqq 1
\end{aligned}
$$

where $p$ is as in equation (1). Thus there exists a positive constant $c_{1}$ such that

$$
\int|v(x, t)|^{p+1} d x \geqq c_{1} t^{-n(p-1) / 2} \quad \text { for all } t \geqq 1 \text {. }
$$

We define

$$
H(t)=\operatorname{Re} \int\left(v_{t} \bar{u}-\bar{v} u_{t}\right) d x
$$


Differentiating $H(t)$ with respect to $t$,

$$
\begin{aligned}
& \frac{d}{d t} H(t)=\operatorname{Re} \int \bar{u}\left(\Delta v-m^{2} v\right)-\bar{v}\left(\Delta u-m^{2} u-u|u|^{p-1}\right) d x \\
&=\left.\left.\operatorname{Re} \int \bar{v} u\right|^{\mid} u\right|^{p-1} d x \\
&=\int|v|^{p+1} d x+\operatorname{Re} \int \bar{v}|u|^{p-1}(u-v) d x \\
& \quad+\int|v|^{2}\left(|u|^{p-1}-|v|^{p-1}\right) d x \\
& \geqq c_{1} t^{-n(p-1) / 2}-I_{1}-I_{2}
\end{aligned}
$$

where

$$
I_{1}=\int|v|\left|u_{\mid}^{\mid p-1}\right| u-\left.\left.v\left|d x, \quad I_{2}=\int\right| v\right|^{2}|| u\right|^{p-1}-|v|^{p-1} \mid d x
$$

Recalling that our free solution $v$ satisfies $\|v(t)\|_{\infty}=o\left(t^{-n / 2}\right)$ as $t \rightarrow+\infty$, first for the special case $p=2, n=1$ or 2 , we have

$$
\begin{aligned}
I_{1} & =\int|v||u \| u-v| d x \leqq \text { const. } t^{-1 / 2}\|u\|_{L^{2}}\|u-v\|_{L^{2}} \\
& =o\left(t^{-n(p-1) / 2}\right) .
\end{aligned}
$$

We now take the general case $1<p \leqq 1+2 n^{-1}$ for $n \geqq 3$ or $1<p<2$ for $n=1$ or 2 . Then, using Hölder's inequality, we have

$$
\begin{aligned}
I_{1} & =\left.\int|v|^{p-1}\left|v_{\mid}^{\prime 2-p}\right| u\right|^{p-1}|u-v| d x \\
& \leqq\|v\|_{\infty}^{p-1}\left(\int|v|^{2} d x\right)^{(2-p) / 2}\left(\int|u|^{2} d x\right)^{(p-1) / 2}\left(\int|v-u|^{2} d x\right)^{1 / 2} \\
& \leqq \text { const. } t^{-n(p-1) / 2}\|u(t)-v(t)\|_{e}=o\left(t^{-n(p-1) / 2}\right) .
\end{aligned}
$$

For $I_{2}$, we have

$$
\begin{aligned}
I_{2} & =\left.\int|v|^{2}|| u\right|^{p-1}-\left.\left.|v|^{p-1}\left|d x=\int\right| v\right|^{p-1}|v|^{3-p}|| u\right|^{p-1}-|v|^{p-1} \mid d x \\
& \leqq\|v\|_{\infty}^{p-1}\left(\int|v|^{2} d x\right)^{(3-p) / 2}\left(\int|u-v|^{2} d x\right)^{(p-1) / 2} \\
& \leqq \text { const. } t^{-n(p-1) / 2}\|u(t)-v(t)\|_{e}=o\left(t^{-n(p-1) / 2}\right) .
\end{aligned}
$$

Thus both $I_{1}$ and $I_{2}$ satisfy the same estimate for sufficiently large $t$, and it 
follows that there is a positive constant $c_{2}$ such that

$$
\frac{d}{d t} H(t) \geqq c_{2} t^{-n(p-1) / 2}
$$

for large enough $t$, say $t \geqq T$. Hence,

$$
H(2 T)-H(T) \geqq c_{2} \int_{T}^{2 T} t^{-n(p-1) / 2} d t \geqq c_{2} \int_{T}^{2 T} t^{-1} d t \geqq c_{2} \log 2>0 .
$$

However, Schwarz's inequality gives

$$
\begin{aligned}
|H(t)| & =\left|\operatorname{Re} \int \bar{u}\left(v_{t}-u_{t}\right)+u_{t}(\bar{u}-\bar{v}) d x\right| \\
& \leqq\|u(t)\|_{e}\|u(t)-v(t)\|_{e} .
\end{aligned}
$$

Thus $|H(t)| \rightarrow 0$ as $t \rightarrow+\infty$, so that $|H(2 T)|+|H(T)| \rightarrow 0$ as $T \rightarrow+\infty$. A sufficiently large choice of $T$ in the inequality above yields the desired contradiction and completes the proof.

Q.E.D.

\section{§ 1. 2. Remarks on Weak Dispersion}

We consider the solutions of the Cauchy problem for (1) with initial data $u(x, 0)=\phi(x), u_{t}(x, 0)=\psi(x)$. If $n=3$, for example, scattering operator is constructed for $p>8 / 3$ and impossible for $1<p \leqq 5 / 3$. For $5 / 3<p \leqq 8 / 3$, we don't know if scattering theory can be constructed, but we can get the following weak result. We denote by $B$ the positive selfadjoint operator $\left(m^{2} I-\Delta\right)^{1 / 2}$ in $L^{2}$, by $\langle,\rangle L^{2}$ inner product, and by $D_{B}$ the domain of $B$ as a Hilbert space relative to the inner product $\langle x, y\rangle_{1}=\langle B x, B y\rangle$. Moreover, we denote by $H$ the Hilbert space direct sum of $D_{B}$ and $L^{2}$ with inner product and norm $\langle,\rangle_{H},|\cdot|_{H}$ and by $\left(\begin{array}{l}x \\ y\end{array}\right)$ the element of $H$ with component $x$ in $D_{B}$ and component $y$ in $L^{2}$. We define

$$
\begin{aligned}
& U(x, t)=\left(\begin{array}{l}
u(x, t) \\
u_{t}(x, t)
\end{array}\right), \quad V(x, t)=\left(\begin{array}{l}
v(x, t) \\
v_{t}(x, t)
\end{array}\right), \quad U_{0}(x)=\left(\begin{array}{l}
\phi(x) \\
\phi(x)
\end{array}\right) \\
& W(t)=\left(\begin{array}{c}
\cos t B, \\
-B \sin t B / B \\
-B, \cos t B
\end{array}\right), \quad K[U(t)]=\left(\begin{array}{c}
0 \\
-|u(t)|^{p-1} u(t)
\end{array}\right) .
\end{aligned}
$$

Then, we have the following 
Theorem 2. Let $u(x, t)$ be $a_{n} \mathcal{E}_{t}^{0}\left(H^{1} \cap L^{2 p}\right) \cap \mathcal{E}_{t}{ }^{1}\left(L^{2}\right)$ solution of the equation (1) with Cauchy data $\phi(x) \in H^{1} \cap L^{2 p}, \psi(x) \in L^{2}$. Suppose that

$$
p>1+2 n^{-1}, n \geqq 3 .
$$

Then there exists a unique free solution $v(x, t) \in \mathcal{E}_{t}{ }^{0}\left(H^{1}\right) \cap \mathcal{E}_{t}{ }^{1}\left(L^{2}\right)$ such that

$$
\langle U(t)-V(t), W(t) X\rangle_{H} \rightarrow 0 \text { as } t \rightarrow+\infty \text {, for any } X \in H \text {. }
$$

Proof. This proof is almost similar to the proof of Theorem 4 in [3]. We note that energy equality (3) is valid also for $u \in \mathcal{E}_{t}^{0}\left(H^{1}\right.$ $\left.\cap L^{2 p}\right) \cap \mathcal{E}_{t}{ }^{1}\left(L^{2}\right)$ and $v \in \mathcal{E}_{t}{ }^{0}\left(H^{1}\right) \cap \mathcal{E}_{t}^{1}\left(L^{2}\right)$ by using Friedrichs' mollifier arguments and that $W(t)$ is the unitary operator on $H$, i.e. $\langle W(t) X, W(t)$ • $Y\rangle_{H}=\langle X, Y\rangle_{H}$ for all $X, Y \in H$. Now $U(t)$ satisfies the integral equation

$$
U(t)=W(t) U_{0}+\int_{0}^{t} W(t-s) K[U(s)] d s .
$$

We define $S(t)=W(-t) U(t)$ and represent by $Z$ an arbitrary fixed element in $D$, where

$$
D=\left\{\left(\begin{array}{c}
x \\
y
\end{array}\right) \mid x \in C_{0}^{\infty}, y \in C_{0}^{\infty}\right\}
$$

From (4), $S(t)$ satisfies

$$
S(t)-S\left(t^{\prime}\right)=\int_{t^{\prime}}^{t} W(-s) K[U(s)] d s .
$$

Then we have

$$
\left\langle S(t)-S\left(t^{\prime}\right), Z\right\rangle_{H}=\int_{t^{\prime}}^{t}\langle K[U(s)], W(s) Z\rangle_{H} d s .
$$

Noting that $W(t) Z$ is a free solution, say of the form $\left(\begin{array}{l}z(t) \\ z_{t}(t)\end{array}\right)$, it follows that

$$
\begin{aligned}
\left|\left\langle S(t)-S\left(t^{\prime}\right), Z\right\rangle_{H}\right| & \leqq\left|\int_{t^{\prime}}^{t}\left\langle-|u(s)|^{p-1} u(s), z_{t}(s)\right\rangle d s\right| \\
& \leqq \int_{t^{\prime}}^{t} \int|u(s)|^{p}\left|z_{t}(s)\right| d x d s .
\end{aligned}
$$


Recalling that $\left\|z_{t}(s)\right\|_{\infty} \leqq$ const. $(1+s)^{-n / 2}$ and (3), it holds that if $p \geqq 2$

$$
\begin{aligned}
\left|\left\langle S(t)-S\left(t^{\prime}\right), Z\right\rangle_{H}\right| & \leqq\left.\int_{t^{\prime}}^{t}\|z(s)\|_{\infty} \int u(s)\right|^{p} d x d s \\
& \leqq \text { const. } \int_{t^{\prime}}^{t}(1+s)^{-n / 2} \int|u(s)|^{2}+|u(s)|^{p+1} d x d s \\
& \leqq \text { const. } \int_{t^{\prime}}^{t}(1+s)^{-n / 2} d s,
\end{aligned}
$$

and if $2>p>1+2 n^{-1}$

$$
\begin{aligned}
\left|\left\langle S(t)-S\left(t^{\prime}\right), Z\right\rangle_{H}\right| & \leqq \int_{t^{\prime}}^{t}\left(\int u^{2} d x\right)^{p / 2}\left(\int\left|z_{t}\right|^{2 /(2-p)} d x\right)^{(2-p) / 2} d s \\
& \leqq \text { const. } \int_{t^{\prime}}^{t}\left\|z_{t}\right\|_{\infty}^{p-1}\left(\int\left|z_{t}\right|^{2} d x\right)^{(2-p) / 2} d s \\
& \leqq \text { const. } \int_{t^{\prime}}^{t}(1+s)^{-n(p-1) / 2} d s .
\end{aligned}
$$

Therefore, if $p>1+2 n^{-1}, n \geqq 3$ right hand sides are integrable, so that

$$
\left\langle S(t)-S\left(t^{\prime}\right), Z\right\rangle_{H} \rightarrow 0 \text { as } t, t^{\prime} \rightarrow+\infty \text {. }
$$

Next, we can show that $\|S(t)\|_{H}$ is bounded because (3) gives

$$
\|S(t)\|_{H}=\|W(-t) U(t)\|_{H}=\|U(t)\|_{H} \leqq \text { const. . }
$$

Now, the fact that $D$ is dense in $H$ and (5) and (6) imply that there exists a unique $S_{0} \in H$ such that

$$
\left\langle S(t)-S_{0}, Z\right\rangle_{H} \rightarrow 0 \text { as } t \rightarrow+\infty \text { for any } Z \in H .
$$

Let $V(t)$ be the free solution given by $V(t)=W(t) S_{0}$. Then for any $Z$ in $H$,

$$
\begin{aligned}
\langle U(t)-V(t), W(t) Z\rangle_{H} & =\langle W(-t) U(t)-W(-t) V(t), Z\rangle_{H} \\
& =\left\langle S(t)-S_{0}, Z\right\rangle \rightarrow 0 \text { as } t \rightarrow+\infty .
\end{aligned}
$$

Finally we show the uniqueness. Suppose that there exist the two different free solutions $V_{1}, V_{2}$ which satisfy the above conditions. From the above arguments, we have

$$
\left\langle V_{1}(t)-V_{2}(t), W(t) Z\right\rangle_{H} \rightarrow 0, t \rightarrow+\infty .
$$

Taking $Z=V_{1}(0)-V_{2}(0)$, 


$$
\left\|V_{1}(t)-V_{2}(t)\right\|_{H} \rightarrow 0, t \rightarrow+\infty .
$$

Since $\left\|V_{1}(t)-V_{2}(t)\right\|_{H}$ is constant, this contradiction implies $V_{1}=V_{2}$.

Q.E.D.

\section{Part II}

\section{§ 2. 1. Solutions of Linear Problem}

We consider the linear equation

(7)

$$
v_{t t}-\Delta v+v_{t}=0 \quad x \in R^{n}, \quad t \geqq 0
$$

with

$$
\left\{\begin{array}{l}
v(x, 0)=\phi(x), \\
v_{t}(x, 0)=\phi(x) .
\end{array}\right.
$$

We can represent the solution of (7) as follows :

$$
v(x, t)=K_{1} * \psi+K_{2} * \phi .
$$

Let $R_{i}(\xi, t)$ be the Fourier transform of $K_{i}(x, t) \quad(i=1,2)$. Then $R_{i}$ satisfies

$$
\begin{aligned}
& \frac{d^{2}}{d t^{2}} R_{i}+\frac{d}{d t} R_{i}+|\xi|^{2} R_{i}=0, \\
& \left\{\begin{array} { l } 
{ R _ { 1 } ( \xi , 0 ) = 0 , } \\
{ \frac { d } { d t } R _ { 1 } ( \xi , 0 ) = 1 , }
\end{array} \quad \left\{\begin{array}{l}
R_{2}(\xi, 0)=1, \\
\frac{d}{d t} R_{2}(\xi, 0)=0 .
\end{array}\right.\right.
\end{aligned}
$$

We can solve (8) exactly, so that

$$
\begin{aligned}
& R_{1}(\xi, t)= \begin{cases}\frac{2 e^{-(1 / 2) t}}{\sqrt{1-4|\xi|^{2}}} \sinh \left(\frac{\sqrt{1-4|\xi|^{2}}}{2} t\right), & |\xi| \leqq \frac{1}{2}, \\
\frac{2 e^{-(1 / 2) t}}{\sqrt{4|\xi|^{2}-1}} \sin \left(\frac{\sqrt{4|\xi|^{2}-1}}{2} t\right), & |\xi|>\frac{1}{2},\end{cases} \\
& R_{2}(\xi, t)=R_{1}(\xi, t)+R_{3}(\xi, t), \\
& R_{3}(\xi, t)= \begin{cases}e^{-(1 / 2) t} \cosh \left(\frac{\sqrt{1-4|\xi|^{2}} t}{2} t\right) & |\xi| \leqq \frac{1}{2} \\
e^{-(1 / 2) t} \cos \left(\frac{\sqrt{4|\xi|^{2}-1}}{2} t\right), & |\xi|>\frac{1}{2}\end{cases}
\end{aligned}
$$


Lemma 1. If $f \in L^{m} \cap H^{[n / 2]+i+|\alpha|}(1 \leqq m \leqq 2)$, then

$$
\begin{aligned}
& \left\|\left(\frac{\partial}{\partial t}\right)^{i}\left(\frac{\partial}{\partial x}\right)^{\alpha}\left(K_{1} * f\right)\right\|_{\infty} \leqq c(1+t)^{-n /(2 m)-i-(1 / 2)|\alpha|}\left(\|f\|_{L^{m}}+\|f\|_{[n / 2]+i+|\alpha|}\right) \\
& \left\|\left(\frac{\partial}{\partial t}\right)^{i}\left(\frac{\partial}{\partial x}\right)^{\alpha}\left(K_{1} * f\right)\right\|_{L_{2}} \leqq c(1+t)^{(n / 4)-n /(2 m)-i-(1 / 2)|\alpha|}\left(\|f\|_{L^{m}}+\|f\|_{i+|\alpha|-1}\right) .
\end{aligned}
$$

If $f \in L^{m} \cap H^{[n / 2]+i+|\alpha|+1}$, then

$$
\begin{aligned}
& \left\|\left(\frac{\partial}{\partial t}\right)^{i}\left(\frac{\partial}{\partial x}\right)^{\alpha}\left(K_{2} * f\right)\right\|_{\infty} \leqq c(1+t)^{-n /(2 m)-i-(1 / 2)|\alpha|}\left(\|f\|_{L^{m}}+\|f\|_{[n / 2]+i+|\alpha|+1}\right) \\
& \left\|\left(\frac{\partial}{\partial t}\right)^{i}\left(\frac{\partial}{\partial x}\right)^{\alpha}\left(K_{2} * f\right)\right\|_{L^{2}} \leqq c(1+t)^{(n / 4)-n /(2 m)-i-(1 / 2)|\alpha|}\left(\|f\|_{L^{m}}+\|f\|_{i+|\alpha|}\right) .
\end{aligned}
$$

Here and hereafter $c$ denotes some constant.

Proof. We note that

$$
\|f\|_{L^{k}} \leqq\|\widehat{f}\|_{L^{m}}, \quad \frac{1}{k}+\frac{1}{m}=1, \quad 1 \leqq m \leqq 2,
$$

where $\widehat{f}$ denotes Fourier transform of $f$. We show lemma 1 only for $\left\|(\partial / \partial t)^{i}(\partial / \partial x)^{\alpha}\left(K_{1} * f\right)\right\|_{\infty}$. For the other cases, we can give proofs in the same way. We use the following

$$
\begin{aligned}
& \int_{0}^{\delta}|\xi|^{k} e^{-c|\xi| 2 t} d|\xi| \leqq c(1+t)^{-(k+1) / 2}, \\
& \sup _{0 \leqq|\xi| \leqq \delta}|\xi|^{k} e^{-c|\xi| 2 t} \leqq c(1+t)^{-k / 2} \quad \text { for all } t \geqq 0
\end{aligned}
$$

which are easily verified. From (9), we have

$$
\begin{aligned}
\left\|\left(\frac{\partial}{\partial t}\right)^{i}\left(\frac{\partial}{\partial x}\right)^{\alpha}\left(K_{1} * f\right)\right\|_{\infty} & \leqq(i \xi)^{\alpha} \cdot \frac{d^{i}}{d t^{i}} R(\xi, t) \cdot \widehat{f}(\xi) \|_{L^{1}} \\
& \leqq c \int|\xi|^{|\alpha|}\left|\frac{d^{i}}{d t^{i}} R(\xi, t) \| \widehat{f}(\xi)\right| d \xi .
\end{aligned}
$$

We take a small fixed $\delta>0$ and divide the last integral into four parts:

$$
\int=\int_{|\xi| \geq 1}+\int_{1 / 2<|\xi|<1}+\int_{\delta \leqq|\xi| \leqq 1 / 2}+\int_{|\xi| \leqq \delta} \equiv I_{1}+I_{2}+I_{3}+I_{4}
$$

We estimate $I_{1} \sim I_{4}$ as follows :

$$
\left|I_{1}\right| \leqq c e^{-(1 / 2) t} \int_{\mid \xi i \geqq 1} \frac{|\xi|^{|\alpha|}\left(1+\sqrt{-1+4|\xi|^{2}}\right)^{i}|\hat{f}(\xi)|}{\sqrt{4|\xi|^{2}-1}} d \xi
$$




$$
\begin{gathered}
\leqq c e^{-t / 2} \sup _{|\xi| \geq 1}\left(\frac{\left(1+\sqrt{4|\xi|^{2}-1}\right)^{i}}{|\xi|^{i-1} \sqrt{\left.4 ! \xi\right|^{2}-1}}\right)\left(\int_{|\xi| \geqq 1}|\xi|^{-2[n / 2]-2} d \xi\right)^{1 / 2} \\
\times\left(\int|\xi|^{2[n / 2]+2|\alpha|+2 i}|\widehat{f}(\xi)|^{2} d \xi\right)^{1 / 2}
\end{gathered}
$$

$\leqq c e^{-t / 2}\|f\|_{[n / 2]+i+|\alpha|}$.

$$
\begin{aligned}
\left|I_{2}\right| & \leqq c e^{-t / 2}\left\{1+\sup _{1>|\xi|>1 / 2}\left(\frac{\sin \left((t / 2) \sqrt{-1+4|\xi|^{2}}\right)}{\sqrt{-1+4|\xi|^{2}}}\right)\right\}\left(\int|f(\xi)|^{2} d \xi\right)^{1 / 2} \\
& \leqq c(1+t) e^{-t / 2}\|f\|_{L^{2}} .
\end{aligned}
$$

$$
\begin{gathered}
\left|I_{3}\right| \leqq c e^{-t / 2}\left\{1+\sup _{\delta<|\xi| \leqq 1 / 2}\left(\frac{\sinh \left((t / 2) \sqrt{1-4|\xi|^{2}}\right)}{\sqrt{1-4|\xi|^{2}}}\right)\right. \\
\left.+\sup _{\delta<|\xi| \leqq 1 / 2} \cosh \left(\frac{t}{2} \sqrt{1-4|\xi|^{2}}\right)\right\}\|f\|_{L^{2}}
\end{gathered}
$$$$
\leqq c e^{-(1 / 2)\left(1-\sqrt{1-4 \delta^{2}}\right) t}\|f\|_{L^{2}} .
$$$$
\left|I_{4}\right| \leqq c \int_{|\xi| \leqq o} \frac{\left(1-\sqrt{1-4|\xi|^{2}}\right)^{i}|\xi|^{|\alpha|}|\widehat{f}(\xi)|}{\sqrt{1-4|\xi|^{2}}} e^{(1 / 2) t\left(-1+\sqrt{1-4|\xi|^{2}}\right)} d \xi
$$$$
+c \int_{|\xi| \leqq \delta} \frac{\left(1+\sqrt{1-4|\xi|^{2}}\right)^{i}|\xi|^{|\alpha|}|\hat{f}(\xi)|}{\sqrt{1-4|\xi|^{2}}} e^{(1 / 2) t\left(-1-\sqrt{1-4|\xi|^{2}}\right)} d \hat{\xi},
$$

since $-4|\xi|^{2} \leqq-1+\sqrt{1-4|\xi|^{2}} \leqq-\triangle_{,-1^{2}}$ for $|\xi|<\frac{1}{2}$,

$$
\begin{aligned}
& \leqq c \int_{|\xi| \leqq \delta}|\xi|^{2 i+|\alpha|} e^{-t|\xi|^{2}}|\widehat{f}(\xi)| d \xi+c e^{-t / 2} \int_{|\xi| \leqq \delta}|\widehat{f}(\xi)| d \hat{\xi} \\
& \leqq c\left(\int_{|\xi| \leqq \delta}|\xi|^{m(2 i+|\alpha|)+n-1} e^{-t|\xi|^{2}} d|\xi|\right)^{1 / m}\left(\int|\widehat{f}(\xi)|^{k} d \xi\right)^{1 / k} \\
& \quad+c e^{-t / 2}\left(\int|\widehat{f}(\xi)|^{k} d \xi\right)^{1 / k} \\
& \quad\left(\frac{1}{m}+\frac{1}{k}=1 \quad 1 \leqq m \leqq 2\right) \\
& \leqq c\left\{(1+t)^{-(n+m(2 i+\mid \alpha()) / 2}\right\}^{1 / m}\|f\|_{L^{m}} \\
& \leqq c(1+t)^{-n /(2 m)-i-(1 / 2)|\alpha|}\|f\|_{L^{m}} .
\end{aligned}
$$

Therefore, from the above estimates, we have

$$
\left\|\left(\frac{\partial}{\partial t}\right)^{i}\left(\frac{\partial}{\partial x}\right)^{\alpha}\left(K_{1} * f\right)\right\|_{\infty} \leqq c(1+t)^{-n /(2 m)-i-(1 / 2)|\alpha|}\left(\|f\|_{L^{m}}+\|f\|_{[n / 2]+i+|a|}\right) .
$$

Q.E.D. 
Lemma 2. We suppose $\phi(x) \in C_{0}{ }^{\infty}, \psi(x) \in C_{0}{ }^{\infty}$. Then the solution of (7) satisfies

$$
\begin{gathered}
\left\|\left(\frac{\partial}{\partial t}\right)^{i}\left(\frac{\partial}{\partial x}\right)^{\alpha} v(t)\right\|_{\infty} \leqq c(1+t)^{-(n / 2)-i-(1 / 2)|\alpha|}\left(\|\phi\|_{[n / 2]+i+|\alpha|+1}+\|\phi\|_{L^{1}}\right. \\
\left.+\|\phi\|_{[n / 2]+i+|\alpha|}+\|\phi\|_{L^{1}}\right) \\
\left\|\left(\frac{\partial}{\partial t}\right)^{i}\left(\frac{\partial}{\partial x}\right)^{\alpha} v(t)\right\|_{L^{2}} \leqq c(1+t)^{-(n / 4)-i-(1 / 2)|\alpha|}\left(\|\phi\|_{i+|\alpha|}+\|\phi\|_{L^{1}}\right. \\
\left.+\|\phi\|_{i+|\alpha|-1}+\|\phi\|_{L^{1}}\right) .
\end{gathered}
$$

The proof of this lemma follows immediately from the lemma 1 taking $m=1$.

\section{§ 2.2. Solutions of Semi-Linear Equations}

We consider the following semi-linear wave equation

$$
\begin{aligned}
& u_{t t}-\Delta u+u_{t}+f\left(u, u_{t}, \nabla u\right)=0, \\
& \left\{\begin{array}{l}
u(x, 0)=\phi(x), \\
u_{t}(x, 0)=\phi(x) .
\end{array}\right.
\end{aligned}
$$

We assume that $f(z)=f\left(z_{1}, z_{2}, \cdots, z_{n+2}\right)$ is $C^{k}$ function on $R^{n+2}$ and satisfies that, for $|z| \leqq 1$,

$$
\begin{aligned}
& |f(z)| \leqq c|z|^{p} \\
& \left|\left(\frac{\partial}{\partial z}\right)^{\alpha} f(z)\right| \leqq c|z|^{p-\mid \alpha_{i}} \quad \text { if } \min (k, p)>|\alpha| \geqq 1, \\
& \left|\left(\frac{\partial}{\partial z}\right)^{\alpha} f(z)\right| \leqq c \quad \text { if } k \geqq|\alpha| \geqq p
\end{aligned}
$$

where $\alpha=\left(\alpha_{1}, \alpha_{2}, \cdots, \alpha_{n+2}\right), p>1$.

As to the estimates of composite function $f(v(x, t))$, we have

Lemma 3. Suppose that $f \in C^{[n / 2]+1+s}(s \geqq[(1+[n / 2]) / 2])$ satisfies (11) and $v(x, t) \in \mathcal{E}_{t}^{0}\left(H^{[n / 2]+1+s}\right)$. Then, $f(v(x, t)) \in \mathcal{E}_{t}^{0}\left(H^{[n / 2]+1+s}\right)$ and satisfies

$$
\begin{array}{rlrl}
\|f(v)\|_{[n / 2]+1+s} \leqq c\|v\|_{c^{s}}^{p-2}\|v\|_{[n / 2]+1+s}^{2} h\left(\|v\|_{[n / 2]+1+s}\right) & & \text { for } p \geqq 2 \\
& \leqq c\|v\|_{[n / 2]+1+s}^{p} h\left(\|v\|_{[n / 2]+1+s}\right) & & \text { for } p>1
\end{array}
$$




$$
\|f(v)\|_{L q} \leqq c\|v\|_{\infty}^{p-(2 / q)}\|v\|_{L^{2}}^{2 / q} h\left(\|v\|_{\infty}\right) \quad \text { for } 1 \leqq q \leqq 2, p q \geqq 2 .
$$

Here and hereafter, we represent by $h(y)$ some nondecreasing nonnegative and continuous function on $y \geqq 0$, and $\|v\|_{c^{s}}$ means $\sum_{|\alpha| \leqq s} \sup \left|(\partial / \partial x)^{\alpha} v(x)\right|$. We omit the proof. (See von Wahl [5]). For the nonlinear term $f$, we consider the three cases

$$
\begin{array}{ll}
\text { case 1. } & f=f\left(u_{t}\right), \\
\text { case 2. } & f=f\left(u_{t}, \nabla u\right), \\
\text { case 3. } & f=f\left(u, u_{t}, \nabla u\right) .
\end{array}
$$

If $n=1$, we have the following

Theorem 3. $(n=1)$ We suppose $\phi(x), \psi(x) \in C_{0}^{\infty}$ and

$$
\|\phi\|_{2+k}+\|\psi\|_{1+k}+\|\phi\|_{L^{1}}+\|\psi\|_{L^{1}} \leqq \varepsilon(k \geqq 0) .
$$

Furthermore, we suppose that $f(z) \in C^{1+k}$ satisfies (11). Then, there exists a small positive constant $\varepsilon_{0}$ such that the Cauchy problem for (2) has a unique $C^{2+k}$ solution for $0<^{\forall} \varepsilon \leqq \varepsilon_{0}$ and $p$ which satisfies the following conditions, and we have

if $p \geqq 2$ for case 1 and 2 or $p>3$ for case 3

$$
\begin{aligned}
& \left\|\left(\frac{\partial}{\partial t}\right)^{i}\left(\frac{\partial}{\partial x}\right)^{j} u(t)\right\|_{L^{2}} \leqq(1+t)^{-\min \{(1 / 4)+i+(1 / 2) j,((1 / 2)+s) p-1 / 2\}} \\
& \text { for } 0 \leqq i+j \leqq 2+k \\
& \left\|\left(\frac{\partial}{\partial t}\right)^{i}\left(\frac{\partial}{\partial x}\right)^{j} u(t)\right\|_{\infty} \leqq c(1+t)^{-\min \{(1 / 2)+i+(1 / 2) j,((1 / 2)+s) p-1 / 2\}} \\
& \text { for } 0 \leqq i+j \leqq 1+k
\end{aligned}
$$

if $2>p>1$ for case 1 , or $2>p>\frac{\sqrt{17}-1}{2}$ for case 2

$$
\begin{aligned}
& \left\|\left(\frac{\partial}{\partial t}\right)^{i}\left(\frac{\partial}{\partial x}\right)^{j} u(t)\right\|_{L^{2}} \leqq c(1+t)^{-\min \{(p-1) / 4+i+(1 / 2) j,((p-1) / 4+s) p\}} \\
& \text { for } 0 \leqq i+j \leqq 2+k \\
& \left\|\left(\frac{\partial}{\partial t}\right)^{i}\left(\frac{\partial}{\partial x}\right)^{j} u(t)\right\|_{\infty} \leqq c(1+t)^{-\min \{(p / 4)+i+(1 / 2) j,((p-1) / 4+s) p\}}
\end{aligned}
$$


for $0 \leqq i+j \leqq 1+k$.

Here, $s$ represents

$$
s=\left\{\begin{array}{l}
1 \text { for case } 1, \\
\frac{1}{2} \text { for case } 2, \\
0 \text { for case } 3
\end{array}\right.
$$

If $n \geqq 2$, we treat only $p \geqq 2$. We have the following

Theorem 4. ( $n \geqq 2)$ We suppose $\phi(x), \psi(x) \in C_{0}^{\infty}$ and

$$
\|\phi\|_{[n / 2]+2+k}+\|\phi\|_{[n / 2]+1+k}+\|\phi\|_{L^{1}}+\|\phi\|_{L^{1}} \leqq \varepsilon, \quad k \geqq\left[\frac{[n / 2]+1}{2}\right] .
$$

Furthermore, we suppose that $f(z) \in C^{[n / 2]+1+k}$ satisfies (11) and $p>2$ for $n=2$ case 3 , or $p \geqq 2$ for the other cases. Then, there exists a small positive constant $\varepsilon_{0}$ such that the Cauchy problem for (2) has a unique $\mathcal{E}_{t}{ }^{0}\left(H^{[n / 2]+3+k}\right) \cap \mathcal{E}_{t}{ }^{1}\left(H^{[n / 2]+2+k}\right)$ solution for $0<^{\forall} \varepsilon \leqq \varepsilon_{0}$ and we have

$$
\begin{aligned}
& \left\|\left(\frac{\partial}{\partial t}\right)^{i}\left(\frac{\partial}{\partial x}\right)^{\alpha} u(t)\right\|_{L^{2}} \leqq c(1+t)^{-\min \{(n / 4)+i+(1 / 2)|\alpha|,((n / 2)+s) p-(n / 2) t} \\
& \text { for } 0 \leqq i+|\alpha| \leqq 3+k+\left[\frac{n}{2}\right] \\
& \left\|\left(\frac{\partial}{\partial t}\right)^{i}\left(\frac{\partial}{\partial x}\right)^{\alpha} u(t)\right\|_{\infty} \leqq c(1+t)^{-\min \{(n / 2)+i+(1 / 2)|\alpha|,((n / 2)+s) p-(n / 2)\}} \\
& \text { for } 0 \leqq i+|\alpha| \leqq 2+k .
\end{aligned}
$$

Here, $s$ represents

$$
s= \begin{cases}1 & \text { for case } 1 \\ \frac{1}{2} & \text { for case } 2, \\ 0 & \text { for case } 3\end{cases}
$$

Proof of Theorem 3. The existence and uniqueness for local solution are well known so that we show decay estimates which show a priori estimates for global solution. Then we suppose the solution $u(x, t) \in C^{2+k}$ exists. $u(x, t)$ satisfies the integral equation 


$$
u(t)=v(t)-\int_{0}^{t} K_{1}(t-\tau) * f\left(u(\tau), u_{t}(\tau), u_{x}(\tau)\right) d \tau,
$$

where $v(t)$ is the solution of linear equation (7) whose Cauchy data are equal to (2). Differentiating (17) with respect to $t$, we have

$$
u_{t}(t)=v_{t}(t)-\int_{0}^{t} \frac{\partial}{\partial t} K_{1}(t-\tau) * f\left(u(\tau), u_{t}(\tau), u_{x}(\tau)\right) d \tau
$$

We only give a proof for the case $1, f=f\left(u_{t}\right)$. For the other cases, we can give proofs by almost the same way. We first note that for $a, b>0$,

$$
\int_{0}^{t}(t-\tau+1)^{-a}(\tau+1)^{-b} d \tau \leqq c(1+t)^{-\min (a, b)}, \quad \max (a, b)>1
$$

that is shown in Segal [4]. From (18) and Lemma 2,

$$
\left\|u_{t}(t)\right\|_{k+1} \leqq c \varepsilon(1+t)^{-5 / 4}+\int_{0}^{t}\left\|\left(\frac{\partial}{\partial t}\right)\left(K_{1} * f\right)\right\|_{k+1} d \tau
$$

Now, we suppose $p \geqq 2$. Then Lemma 1 with $m=1$ gives

$$
\begin{array}{r}
\left\|u_{t}(t)\right\|_{k+1} \leqq c \varepsilon(1+t)^{-5 / 4}+c \int_{0}^{t}(1+t-\tau)^{-5 / 4}\left\{\left\|f\left(u_{t}(\tau)\right)\right\|_{L^{1}}\right. \\
\left.+\left\|f\left(u_{t}(\tau)\right)\right\|_{k+1}\right\} d \tau .
\end{array}
$$

Lemma 3 gives

$$
\left\|f\left(u_{t}\right)\right\|_{L^{1}}+\left\|f\left(u_{t}\right)\right\|_{k+1} \leqq c\left\|u_{t}\right\|_{c^{k}}^{p-2}\left\|u_{t}\right\|_{k+1}^{2} h\left(\left\|u_{t}\right\|_{k+1}\right) .
$$

Substituting the above into (19),

$$
\left\|u_{t}(t)\right\|_{k+1} \leqq c \varepsilon(1+t)^{-5 / 4}+c \int_{0}^{t}(1+t-\tau)^{-5 / 4}\left\|u_{t}(\tau)\right\|_{k+1}^{p} h\left(\left\|u_{t}(\tau)\right\|_{k+1}\right) d \tau
$$

putting $M(t)=\sup _{0 \leqq r \leqq t}(1+\tau)^{5 / 4}\left\|u_{t}(\tau)\right\|_{k_{-1}}$,

$$
\begin{aligned}
& \leqq c \varepsilon(1+t)^{-5 / 4}+c h(M(t)) \int_{0}^{t}(1+t-\tau)^{-5 / 4}(1+\tau)^{-(5 / 4) p}(M(\tau))^{p} d \tau \\
& \leqq c \varepsilon(1+t)^{-5 / 4}+c(1+t)^{-5 / 4}(M(t))^{p} h(M(t))
\end{aligned}
$$

so that

$$
M(t) \leqq c \varepsilon+c(M(t))^{p} h(M(t)) .
$$

Therefore, there exists $\varepsilon_{0}$ such that $M(t) \leqq c$ for $0<\varepsilon \leqq \varepsilon_{0}$ (cf [4]) so that

$$
\left\|u_{t}(t)\right\|_{k+1} \leqq c(1+t)^{-s / 4} .
$$

Next, from (18) and Lemmas 1,2 and 3, 


$$
\begin{aligned}
\left\|u_{t}(t)\right\|_{c k} \leqq c(1+t)^{-3 / 2}+c \int_{0}^{t}(1+t-\tau)^{-3 / 2}\left\|u_{t}(\tau)\right\|_{k+1}^{p} h\left(\left\|u_{t}(\tau)\right\|_{k+1}\right) d \tau \\
\leqq c(1+t)^{-3 / 2}+c \int_{0}^{t}(1+t-\tau)^{-3 / 2}(1+\tau)^{-(5 / 4) p} d \tau
\end{aligned}
$$

Hence,

$$
\left\|u_{t}(t)\right\|_{c^{k}} \leqq c(1+t)^{-3 / 2} .
$$

Furthermore, for $i=0,1$,

(22) $\left(\frac{\partial}{\partial t}\right)^{i}\left(\frac{\partial}{\partial x}\right)^{j} u=\left(\frac{\partial}{\partial t}\right)^{i}\left(\frac{\partial}{\partial x}\right)^{j} v-\int_{0}^{t}\left(\frac{\partial}{\partial t}\right)^{i}\left(\frac{\partial}{\partial x}\right)^{j}\left(K_{1} * f\right) d \tau$.

By Lemma 2, we have

$$
\begin{aligned}
& \left\|\left(\frac{\partial}{\partial t}\right)^{i}\left(\frac{\partial}{\partial x}\right)^{j} v(t)\right\|_{L^{2}} \leqq c(1+t)^{-(1 / 4)-i-(1 / 2) j}, \\
& \left\|\left(\frac{\partial}{\partial t}\right)^{i}\left(\frac{\partial}{\partial x}\right)^{j} v(t)\right\|_{\infty} \leqq c(1+t)^{-(1 / 2)-i-(1 / 2) j} .
\end{aligned}
$$

By virtue of Lemmas 1 and 3, we have

$$
\begin{aligned}
& \int_{0}^{t}\left\|\left(\frac{\partial}{\partial t}\right)^{i}\left(\frac{\partial}{\partial x}\right)^{j} K_{1} * f\left(u_{t}(\tau)\right)\right\|_{L^{2}} d \tau \\
& \quad \leqq c \int_{0}^{t}(1+t-\tau)^{-(1 / 4)-i-(1 / 2) j}\left\|u_{t}(\tau)\right\|_{c^{k}}^{p-2}\left\|u_{t}(\tau)\right\|_{k+1}^{2} d \tau
\end{aligned}
$$

substituting (20) and (21) into the above,

$$
\begin{aligned}
& \leqq c \int_{0}^{t}(1+t-\tau)^{-(1 / 4)-i-(1 / 2) j}(1+\tau)^{-(3 / 2) p+1 / 2} d \tau \\
& \leqq c(1+t)^{-\min ((1 / 4)+i+(1 / 2) j,(3 / 2) p-1 / 2)} \\
& \text { for } 0 \leqq i+j \leqq k+2 .
\end{aligned}
$$

Analogously, we have

$$
\begin{gathered}
\int_{0}^{t}\left\|\left(\frac{\partial}{\partial t}\right)^{i}\left(\frac{\partial}{\partial x}\right)^{j} K_{1} * f\left(u_{t}(\tau)\right)\right\|_{\infty} d \tau \\
\leqq c(1+t)^{-\min ((1 / 2)+i+(1 / 2) j,(3 / 2) p-1 / 2)} \\
\quad \text { for } \quad 0 \leqq i+j \leqq k+1
\end{gathered}
$$

Thus, we can get the desired estimates (13) and (14) for $i=0,1$. For 
$i \geqq 2$, we have

$$
\begin{aligned}
& \left(\frac{\partial}{\partial t}\right)^{i}\left(\frac{\partial}{\partial x}\right)^{j} u=\text { right hand side of }(22) \\
& -\sum_{s=0}^{i-2}\left(\frac{\partial}{\partial t}\right)^{s}\left(\frac{\partial}{\partial x}\right)^{j}\left\{\left(\frac{\partial}{\partial t}\right)^{i-1-s} K_{1}(0) * f\left(u_{t}(t)\right)\right\} .
\end{aligned}
$$

Our problem is the last term. For the moment, we suppose that

$$
\begin{aligned}
& \sum_{s=1}^{i}\left\|\left(\frac{\partial}{\partial t}\right)^{s} u\right\|_{k+1-s} \leqq c(1+t)^{-5 / 4}, \\
& \sum_{s=1}^{i}\left\|\left(\frac{\partial}{\partial t}\right)^{s} u\right\|_{c^{k+1-s}} \leqq c(1+t)^{-3 / 2} \\
& \text { for } 0 \leqq i \leqq i_{0} .
\end{aligned}
$$

Then, let's show (13) and (14) for $i=i_{0}+1$. From (8), it follows

$$
\left(\frac{d}{d t}\right)^{s+2} R_{1}(\xi, 0)=\sum_{n=1}^{s+1} \lambda_{1}{ }^{m} \lambda_{2}{ }^{s+1-m}, \quad \text { where } \lambda_{1,2}=\frac{-1 \pm \sqrt{1-4|\xi|^{2}}}{2} .
$$

Hence, from (23) with $i=i_{0}+1$, we have

$$
\begin{array}{r}
\sum_{s=0}^{i_{0}-1}\left\|\left(\frac{\partial}{\partial t}\right)^{s}\left(\frac{\partial}{\partial x}\right)^{j}\left\{\left(\frac{\partial}{\partial t}\right)^{i-1-s} K_{1}(0) * f\left(u_{t}(t)\right)\right\}\right\|_{L^{2}} \\
\leqq c \sum_{s=0}^{i_{0}-1}\left\|\left(\frac{\partial}{\partial t}\right)^{s} f\left(u_{t}\right)\right\|_{i+j-2-s} \\
\leqq c\left(\sum_{s=1}^{i_{0}}\left\|\left(\frac{\partial}{\partial t}\right)^{s} u\right\|_{c^{k+1-s}}\right)^{p-1}\left(\sum_{s=1}^{i_{0}}\left\|\left(\frac{\partial}{\partial t}\right)^{s} u\right\|_{k+2-s}\right) \\
\times h\left(\sum_{s=1}^{i_{0}}\left\|\left(\frac{\partial}{\partial t}\right)^{s} u\right\|_{c^{k+1-s}}\right)
\end{array}
$$

substituting (24) into the above

$$
\leqq c(1+t)^{-(3 / 2) p+1 / 4} \leqq c(1+t)^{-\min ((1 / 4)+i+(1 / 2) j,(3 / 2) p-(1 / 2))} .
$$

In the same way, we have

$$
\begin{gathered}
\sum_{s=0}^{i-2}\left\|\left(\frac{\partial}{\partial t}\right)^{s}\left(\frac{\partial}{\partial x}\right)^{j}\left\{\left(\frac{\partial}{\partial t}\right)^{i-1-s} K_{1}(0)^{*} f\left(u_{t}(t)\right)\right\}\right\|_{\infty} \\
\leqq c(1+t)^{-\min ((1 / 2)-i+(1 / 2) j,(3 / 2) p-(1 / 2))} .
\end{gathered}
$$

Thus, (13) and (14) hold for $i=i_{0}+1$ and this clearly shows that (24) 
holds for $i=i_{0}+1$. Therefore, we can get the desired decay estimates inductively because (24) is true for $i=0,1$ as we verified already.

If $1<p<2$, Lemmas 1 and 2 give

$$
\begin{gathered}
\left\|u_{t}(t)\right\|_{k+1} \leqq c \varepsilon(1+t)^{-(5 / 4)}+c \int_{0}^{t}(1+t-\tau)^{-(3 / 4)-(1 / 2 m)}\left(\left\|f\left(u_{t}\right)\right\|_{L^{m}}\right. \\
\left.+\left\|f\left(u_{t}\right)\right\|_{k+1}\right) d \tau .
\end{gathered}
$$

Then choosing $m$ as $p m=2$ in Lemma 3 ,

$$
\leqq c \varepsilon(1+t)^{-5 / 4}+c \int_{0}^{t}(1+t-\tau)^{-(p+3) / 4}\left\|u_{t}(\tau)\right\|_{k+1}^{p} h\left(\left\|u_{t}(\tau)\right\|_{k+1}\right) d \tau .
$$

We can get

$$
\left\|u_{t}(t)\right\|_{k+1} \leqq c(1+t)^{-(p+3) / 4}, \quad 0<\varepsilon \leqq \varepsilon_{0}
$$

by the same method as before. The remainder is same as $p \geqq 2$.

Q.E.D.

We omit the proof of the Theorem 4 because we show it by the same way as in the Theorem 3 .

\section{§ 2. 3. Special Case $f=\left|u_{t}\right|^{p-1} u_{t}, n=1$.}

We consider the following equation

$$
u_{t t}-u_{x x}+u_{t}+\left|u_{t}\right|^{p-1} u_{t}=0, x \in R^{1}, p>1
$$

with

$$
\left\{\begin{array}{l}
u(x, 0)=\phi(x) \in C_{0}^{\infty}, \\
u_{t}(x, 0)=\phi(x) \in C_{0}^{\infty} .
\end{array}\right.
$$

For this case, we can get the decay estimates without the smallness conditions of $\phi$ and $\psi$. We first prepare the following

Lemma 4. Let $u(x, t)$ be a $C_{0}^{2}$ solution of the Cauchy problem for (25). Then

$$
\left\|u_{t}(t)\right\|_{\infty},\left\|u_{t}(t)\right\|_{L^{2}} \rightarrow 0 \text { as } t \rightarrow+\infty .
$$

Proof. From (21), we have

$$
\text { (26) } \int_{0}^{t} \int u_{t}{ }^{2}(\tau) d x d \tau \leqq E, \int\left(u_{t}{ }^{2}(t)+u_{t t}^{2}(t)+u_{t . x}^{2}(t)\right) d x \leqq E \text { for } t \geqq 0
$$


where $E$ is some constant that depends only on $\phi$ and $\psi$ From (26), we have

$$
\begin{aligned}
\left|\left(\int u_{t}{ }^{2}\left(t_{1}\right) d x\right)^{2}-\left(\int u_{t}{ }^{2}\left(t_{2}\right) d x\right)^{2}\right| & =\left|4 \int_{t_{2}}^{t_{1}}\left(\int u_{t}{ }^{2} d x\right)\left(\int u_{t} u_{t} d x\right) d \tau\right| \\
& \leqq E \mid \int_{t_{2}}^{t_{1}} \int u_{t}{ }^{2}(\tau) d x d \tau
\end{aligned}
$$

Therefore, $\left\|u_{t}(t)\right\|_{L^{2}}$ is asymptotic to constant as $t \rightarrow+\infty$. If this constant is not zero, it contradicts (26). Thus we have

$$
\left\|u_{t}(t)\right\|_{L^{2}} \rightarrow 0 \text { as } t \rightarrow+\infty \text {. }
$$

Furthermore, from

$$
u_{t}^{2}(t)=\int_{-\infty}^{t} 2 u_{t} u_{t . x} d x \leqq 2\left\|u_{t}(t)\right\|_{L^{2}}\left\|u_{t x}(t)\right\|_{L^{2}}
$$

we have

$$
\left\|u_{t}(t)\right\|_{L^{2}} \rightarrow 0 \text { as } t \rightarrow+\infty \text {. }
$$

Q.E.D.

Thcorem 5. We suppose that $\phi(x) \in C_{0}^{\infty}, \dot{\psi}(x) \in C_{0}^{\infty}$ and $p>1$. Then, the Cauchy problem for (25) has a unique $C^{2}$ solution such that; if $p \geqq 2$

$$
\begin{aligned}
& \left\|\left(\frac{\partial}{\partial t}\right)^{i}\left(\frac{\partial}{\partial x}\right)^{j} u(t)\right\|_{L^{2}} \leqq c(1+t)^{-(1 / 4)-i-(1 / 2) j} \text { for } i+j \leqq 2 \\
& \left\|\left(\frac{\partial}{\partial t}\right)^{i}\left(\frac{\partial}{\partial x}\right)^{j} u(t)\right\|_{\infty} \leqq c(1+t)^{-(1 / 2)-i-(1 / 2) j} \text { for } i+j \leqq 1
\end{aligned}
$$

and if $p<2$

$$
\begin{aligned}
& \left\|\left(\frac{\partial}{\partial t}\right)^{i}\left(\frac{\partial}{\partial x}\right)^{i} u(t)\right\|_{L^{2}} \leqq c(1+t)^{-\min \left\{(p-1) / 4+i_{\top}(1 / 2) j, p(p+3) / 4\right\}} \text { for } i+j \leqq 2 \\
& \left\|\left(\frac{\partial}{\partial t}\right)^{i}\left(\frac{\partial}{\partial x}\right)^{j} u(t)\right\|_{\infty} \leqq c(1+t)^{-\min \{(p / 4)+i+(1 / 2) j, p(p+3) / 4\}} \text { for } i+j \leqq 1
\end{aligned}
$$

Proof. We only give a proof for $p \geqq 2$ because the reasoning for $p<2$ is almost the same. (18) and Lemmas 1 and 2 give

$$
\left\|u_{t}(t)\right\|_{1} \leqq c(1+t)^{-(5 / 4)}+c \int_{0}^{t}(1+t-\tau)^{-(5 / 4)}\left\{\left\|\left|u_{t}\right|^{p-1} u_{t}\right\|_{1}\right.
$$


$\left.+\left\|\left|u_{t}\right|^{p-1} u_{t}\right\|_{L^{1}}\right\} d \tau$

For this case, we use

$$
\left\||v|^{p-1} v\right\|_{1} \leqq c\|v\|_{\infty}^{p-1}\|v\|_{1},\left\||v|^{p-1} v\right\|_{L^{1}} \leqq\|v\|_{\infty}^{p=2}\|v\|_{L^{1}}^{2}
$$

that are easily verified by the modified lemma 3 . Therefore, we have

$$
\left\|u_{t}(t)\right\|_{1} \leq c(1+t)^{-5 / 4}+c I(0, t)
$$

where

$$
I(0, t)=\int_{0}^{t}(1+t-\tau)^{-5 / 4}\left(\left\|u_{t}(\tau)\right\|_{L^{2}}+\left\|u_{t}(\tau)\right\|_{\infty}\right)\left\|u_{t}(\tau)\right\|_{1}\left\|u_{t}(\tau)\right\|_{\infty}^{p-2} d \tau .
$$

Now, we divide $I(0, t)$ into $I(0, t / 2)+I(t / 2, t)$ and define $M(t)$ by

$$
M(t)=\sup _{0 \leqq t \leqq t}(1+\tau)^{5 / 4}\left\|u_{t}(\tau)\right\|_{1}
$$

It follows that

$$
\begin{aligned}
& I\left(0, \frac{1}{2} t\right) \leqq c \int_{0}^{(1 / 2) t}(1+t-\tau)^{-5 / 4}\left\|u_{t}(\tau)\right\|_{1} d \tau \\
& \leqq c\left(1+\frac{t}{2}\right)^{-5 / 4} \int_{0}^{(1 / 2) t}(1+\tau)^{-5 / 4} M(\tau) d \tau, \\
& I\left(\frac{1}{2} t, t\right) \leqq \sup _{(1 / 2) t \leqq \tau \leqq t}\left\{\left\|u_{t}\right\|_{\infty}^{p-1}+\left\|u_{t}\right\|_{\infty}^{p-2}\left\|u_{t}\right\|_{L^{2}}\right\} \\
& \times \int_{(1 / 2) t}^{t}(1+t-\tau)^{-5 / 4}(1+\tau)^{-5 / 4} M(\tau) d \tau \\
& \leqq c\left(1+\frac{t}{2}\right)^{-5 / 4} M(t) \sup _{(1 / 2) t \leqq \tau \leqq t}\left\{\left\|u_{t}(\tau)\right\|_{\infty}^{p-1}+\left\|u_{t}(\tau)\right\|_{\infty}^{p-2}\left\|u_{t}(\tau)\right\|_{\left.L_{2}\right\}}\right\}
\end{aligned}
$$

From the above estimates, we have

$$
\begin{aligned}
M(t) \leqq & +c \int_{0}^{(1 / 2) t}(1+\tau)^{-5 / 4} M(\tau) d \tau \\
& +c \sup _{(1 / 2) t \leqq \tau \leqq t}\left\{\left\|u_{t}(\tau)\right\|_{\infty}^{p-1}+\left\|u_{t}(\tau)\right\|_{\infty}^{p-2}\left\|u_{t}(\tau)\right\|_{L^{2}}\right\} M(t) .
\end{aligned}
$$

Then, by Lemma 4 , the choice of sufficient large $t$, say $t \geqq T$, gives

$$
c \sup _{(1 / 2) t \leqq \tau \leqq t}\left\{\|u(\tau)\|_{\infty}^{p-1}+\|u(\tau)\|_{\infty}^{p-2}\|u(\tau)\|_{L^{2}}\right\} \leqq \frac{1}{4} .
$$

Therefore, we have 


$$
M(t) \leqq c+c \int_{0}^{t}(1+\tau)^{-5 / 4} M(\tau) d \tau \quad \text { for all } t \geqq T
$$

This implies

$$
\left\|u_{t}(t)\right\|_{1} \leqq c(1+t)^{-5 / 4},
$$

so that we can get the conclusion by the same way as in Theorem 3 .

Q.E.D.

The author wishes to express his sincere gratitude to Professor Yujiro Ohya and Doctor Takaki Nishida for their many suggestions and criticisms.

\section{References}

[1] Glassey, R. T., On the asymptotic behavior of nonlinear wave equation, Trans. Amer. Math. Soc., 182 (1973), 187-200.

[2] Morawetz, C. S. and Strauss, W. A., Decay and scattering of solutions of a nonlinear relativistic wave equation, Comm. Pure Appl. Math., 25 (1972), 1-31.

[3] Segal, I. E., Quantization and dispersion for nonlinear relativistic equations, Mathematical Theory of Elementary Particles, M.I.T. Press, Cambridge, Mass., 1966, 79-108.

[4] - Dispersion for non-linear relativistic equations, II, Ann. Sci. École Norm. Sup., (4) 1 (1968), 459-497.

[5] von Wahl, W., Über die klassische Lösbarkeit des Cauchy-Problems für nichtlineare Wellengleichungen bei kleinen Anfangswerten und das asymptotische Verhalten der Lösungen, Math. Z., 114 (1970), 281-299. 
